\title{
Performance Evaluation of CTP Routing Protocol for Mobile Wireless Sensor Network
}

\author{
Mohamad Nazim Jambli*, Azlina A. J.*, Farha Anati A. M.*, Halikul Lenando*, Johari Abdullah*, Sinarwati M. S. ${ }^{\dagger}$ \\ *Faculty of Computer Science and Information Technology, Universiti Malaysia Sarawak, 94300 Sarawak, Malaysia \\ \{jmnazim, ajazlina, farhamansor, cool, ajohari\}@ fit.unimas.my \\ $\dagger$ Centre for Pre-University Studies, Universiti Malaysia Sarawak, 94300 Sarawak, Malaysia \\ mssinarwati@preuni.unimas.my
}

\begin{abstract}
Recently, extensive research has been conducted on Wireless Sensor Network (WSNs) due to their wide range of potential applications ranging from environmental monitoring to critical military surveillance. Most of these applications can be deployed either in static or mobile environment. In static WSN, the change of sensor nodes' topology is normally caused by node failure which is due to energy depletion. However, in mobile WSN (MWSN), the main reason of the topology change is caused by the node movement. Since the sensor nodes are limited in power supply and have a low radio frequency coverage, they are easily losing their connection with neighbours and difficulties to transmit their packets towards sink node. The reconnection process from one node to another node consumes more energy that related to control packets. Using Collection Tree Protocol (CTP) routing protocol in MWSN application shows degradation in network performance due to high speed of mobile nodes. In this paper, through extensive simulation we evaluated the capability of CTP on how far it can react to network topology change in MWSN. We investigated the performance metrics namely data delivery ratio (DDR) and energy consumption of mobile nodes with various speed. Our performance study demonstrates that by applying the existing CTP in MWSN, the results show a low percentage of data delivery ratio and higher total network energy consumption in high speed of mobile nodes due to serious broken link and frequent tree re-generation caused by nodes movement. We also identify factors that contribute to the degradation of CTP's performance and highlight some key research problems that need to be addressed for successful implementation of CTP in MWSN.
\end{abstract}

Index Terms-Mobile Wireless Sensor Network, Collection Tree Protocol, Performance Evaluation.

\section{INTRODUCTION}

In recent years, extensive research has been conducted on Wireless Sensor Networks (WSNs) due to their wide range of potential applications. The enormous potential of this technology can be seen ranging from environmental monitoring to critical military surveillance and healthcare applications [1]. In these networks a large number of small sensor nodes are deployed, each capable of collecting, storing, processing observations and communicating over short-range wireless interfaces and multiple hops to central locations called sinks. However, the nodes in WSNs have severe resource constraints due to their lack of processing power, limited memory, bandwidth and energy [?]. Since these networks are usually deployed in remote places and left unattended, they should be equipped with energy-constraint routing protocol to increase the network lifetime.

The researchers in WSNs have proposed various routing protocols which are optimized for these networks with re- source constraints. A number of efficient routing protocols has been proposed by several researchers in WSNs [2], [3]. When describing the existing routing protocols, normally they assumed that sensor nodes and sinks are stationary in the deployment. But for some WSN applications, a mobile node is also present because a mobile Wireless Sensor Network (MWSN) owes its name to the presence of mobile sink or sensor nodes within the network [4]. For example in ocean temperature monitoring application, the sensors are deploy on the surface of the ocean to monitor the water temperature and we can expect that they are carried around by ocean flows [5]. As for a real-time target tracking in battle situations, mobile nodes can be used to avoid holes in the coverage and to generate information to be transmitted through the network[6]. In some other applications, sensors are mounted on robots, animals or other moving objects, which can sense and collect relevant information. If this information is not managed properly, energy can be wasted due to unpredictable changes in network topology in MWSN.

In WSN, there is numerous number of data collection protocol that has been introduced and used. Among all of these, Collection Tree Protocol (CTP) is widely used as the reference protocol for data collection and also has large number of applications [7]. Increasing interest in mobile WSN application give a challenge in designing efficient WSN as the sensor nodes has severe constraint in limited power source, memory, bandwidth and energy. In mobile WSN, mobile nodes are deployed and these moving nodes network make network topology keep changing. Thus, the researchers and designers have to think about every aspect that can make the network lifetime is last longer. So, they proposed several routing protocol for such network that can transmit the data correctly and reliably from a mobile nodes to the sink node.

Since the sensors are limited in power supply, they have a low radio frequency coverage. This can be disadvantageous to mobile sensor nodes because they easily lose their connection with neighbours as indicated in [8], and as a consequence, they have difficulties in updating their routing tables. The switching process from one area to another consumes more energy that related to transmitting and receiving control packets. These are the primary problems that need to be addressed in MWSN routing protocol. Thus, in this paper, through simulation we evaluated the capability of CTP on how far it can react to network topology change in MWSN. We investigated the performance metrics namely data delivery ratio (DDR) and 